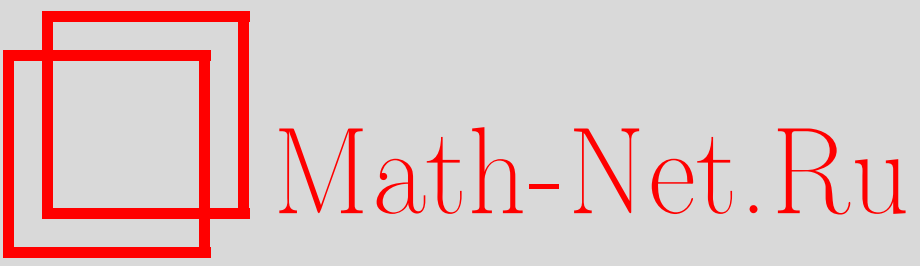

В. Е. Зобов, Особые точки зависящих от времени корреляционных функций спиновых систем на решетках большой размерности при высоких температурах, ТМФ, 2000, том 123, номер 1, 116-131

DOI: https://doi.org/10.4213/tmf591

Использование Общероссийского математического портала Math-Net.Ru подразумевает, что вы прочитали и согласны с пользовательским соглашением

http://www.mathnet.ru/rus/agreement

Параметры загрузки:

IP : 54.162 .27 .143

26 апреля 2023 г., $17: 59: 08$ 


\section{ОСОБЫЕ ТОЧКИ ЗАВИСЯШИХ ОТ ВРЕМЕНИ КОРРЕЛЯЦИОННЫХ ФУНКЦИЙ СПИНОВЫХ СИСТЕМ НА РЕШЕТКАХ БОЛЬШОЙ РАЗМЕРНОСТИ ПРИ ВЫСОКИХ ТЕМПЕРАТУРАХ}

Исследованы зависящие от времени автокорреляционные функции гейзенберговской модели со спинами $1 / 2$ на $d$-мерных простых кубических решетках при больших размерностях $d$ и бесконечной температуре. Автокорреляционная функция на оси мнимого времени интерпретируется как производящая функция нагруженных деревьев, построенных с двойными связями. Эти деревья дают главные члены по $1 / d$ для коэффициентов временного разложения автокорреляционной функции. К производящей функции приближения Бете для таких деревьев выведены поправочные члены от пересечения ветвей. Предложена процедура определения по ним поправки к координате особой точки производящей функции (величине, обратной параметру роста деревьев) без расчета числа деревьев. Найдены имеющие порядок величины $1 / \sigma^{2}$ (где $\sigma=2 d-1$ ) главные поправки к координатам особых точек излучаемой автокорреляционной функции и производящей функции для деревьев в модели Идена, построенных с одинарными связями.

\section{1. ВВЕДЕНИЕ}

Обшепризнана и всесторонне изучена сильная зависимость от размерности пространства $d$ характеристик сингулярностей, описывающих фазовые переходы и другие критические явления. Значительно менее изучена зависимость от $d$ аналитических свойств динамических корреляционных функций квантовых спиновых систем при высоких температурах. Было доказано [1], что у этих функций в одномерном случае нет особых точек на конечном расстоянии в плоскости комплексной временной переменной. С другой стороны, в работе [2] было установлено, что автокорреляционная функция гейзенберговского магнетика на решетке бесконечной размерности при бесконечной температуре имеет особые точки на оси мнимого времени на конечном расстоянии от начала координат. Для систем произвольной размерности $d$ строгий результат не известен.

Мощным методом исследования особенностей функций является метод степенных рядов. K таким рядам относятся высокотемпературные разложения в теории фазовых пе-

${ }^{*}$ Институт физики им. Л.В. Киренского Сибирского отделения РАН, Красноярск, Академгородок, Россия. E-mail:root@iph.krasnoyarsk.su 
реходов, производящие функции для кластеров (решеточных “зверей” или фигур) в теории полимеров и перколяции и, наконец, ряды по степеням времени спиновых корреляционных функций, рассматриваемые в данной работе. Коэффициенты в этих рядах или, что то же самое, спектральные моменты можно представить в виде суммы нагруженных решеточных фигур $[3,4]$. В пределе $d \rightarrow \infty$ в этой сумме остаются деревья, построенные на решетке с помошью двойных связей $[2,4]$, а корреляционную функцию на оси мнимого времени можно трактовать как производящую функцию для таких деревьев. В отличие от деревьев теории полимеров и перколяции $[5,6]$ весовой множитель включает число способов построения данного дерева. Подобным свойством обладают деревья в модели Идена [7], построенные с одинарными связями и поэтому более простые.

При переходе к спиновым системам на гиперкубических решетках большой, но конечной размерности вклад в моменты от деревьев с двойными связями не является единственным, тем не менее все еше оставаясь главным. Представление временных корреляционных функций такими деревьями позволяет исследовать возможные изменения аналитических свойств этих функций при изменении размерности пространства. Прежде всего при уменьшении размерности $d$ появляется ограничение по числу ветвей, выходящих из одного узла дерева. Соответствуюшее увеличение координаты ближайшей особой точки автокорреляционной функции найдено в работе [4] в приближении Бете, применявшемся ранее к деревьям с одинарными связями $[5,7]$. Другим следствием конечности $d$ является усиление влияния пересечения ветвей деревьев (эффект исключенного объема). В статье [4] этот эффект изучался методом компюютерного моделирования для двух- и трехмерных решеток. На решетках большой размерности для учета подобных эффектов применяется метод разложения по обратной размерности пространства $(1 / d$ или $1 / \sigma$, где $\sigma=2 d-1)[6,8-10]$. В теории полимеров получено несколько членов $1 / \sigma$-разложения для параметров роста решеточных фигур и деревьев $[6,9,10]$, т.е. для величин, обратных координатам особых точек производяших функций. В данной работе найдены имеющие порядок $1 / \sigma^{2}$ главные поправки к координатам ближайших особых точек указанной вьше автокорреляционной функции и производяшей функции деревьев в модели Идена. В модели Идена ранее были рассчитаны поправки $1 / d$ к среднеквадратичному радиусу кластера $[11,12]$. Статей с поправками к параметру роста кластеров нам не известно.

Во втором разделе данной работы описывается рассматриваемая спиновая модель и формулируется комбинаторная задача. В разделе 3 получены формулы для поправки от пересечения ветвей к производящей функции для деревьев, построенных с двойными связями. На основании этих формул в разделе 4 рассчитываются поправки к координатам ближайших особых точек производящих функций. В первой части этого раздела расчет выполнен для деревьев, построенных с одинарными связями, а во второй - с двойными. В приложении предложенный подход проверяется на решеточных фигурах.

\section{2. МОДЕЛЬ}

Рассмотрим зависящую от времени автокорреляционную функцию спина, расположенного в одном из узлов простой гиперкубической решетки размерности $d$, при беско- 
нечной температуре

$$
F(t)=\frac{\operatorname{Sp}\left\{\exp (i \varkappa t) S_{0}^{\alpha} \exp (-i \varkappa t) S_{0}^{\alpha}\right\}}{\operatorname{Sp}\left\{\left(S_{0}^{\alpha}\right)^{2}\right\}},
$$

где $\varkappa$ - гамильтониан изотропной модели Гейзенберга со взаимодействием $J$ между ближайшими соседями, $S_{0}^{\alpha}-\alpha$-компонента $(\alpha=x, y, z)$ векторного оператора спина в узле $0, S=1 / 2$. Функция (2.1) может быть разложена в степенной ряд

$$
F(t)=\sum_{n=0}^{\infty} \frac{(-1)^{n}}{(2 n) !} M_{2 n} t^{2 n}
$$

где $n$-й коэффициент разложения определяется через $2 n$-кратный коммутатор

$$
M_{2 n}=\frac{\operatorname{Sp}\left\{\left[\varkappa,\left[\varkappa, \ldots\left[\varkappa, S_{0}^{\alpha}\right] \ldots\right] S_{0}^{\alpha}\right]\right\}}{\operatorname{Sp}\left\{\left(S_{0}^{\alpha}\right)^{2}\right\}} .
$$

Как известно, $M_{2 n}$ является моментом порядка $2 n$ спектральной плотности автокорреляционной функции (2.1).

Правила вычисления многократных коммутаторов в (2.3) подробно разбираются, например, в работах $[2,3,13-16]$. С геометрической точки зрения они имеют много общего со случаем процесса роста полимерной молекулы или кластера $[7,17]$. Действительно, если каждой паре взаимодействующих спинов в гамильтониане ставить в соответствие связь на решетке между узлами, занимаемыми этими спинами, то каждая коммутация добавляет к уже построенному кластеру связь. Известные свойства матриц Паули приводят к следующим результатам. Кластер строится из связей. В его узлах располагаются операторы $S_{i}^{\alpha}$ или $\left(S_{i}^{\alpha}\right)^{2}=1 / 4$. Будем называть первые узлы активными, а вторые - неактивными. Каждая коммутация добавляет новую связь к одному из активных узлов кластера. Если свободный конец связи попадает в незанятый узел решетки, то кластер увеличивается на один активный узел. Если же свободный конец связи попадает на уже присоединенный к кластеру узел, то результат коммутации зависит от проекции спинового оператора этого узла. Этот результат может оказаться нулевым или выразиться в изменении активности рассматриваемого узла: активный узел становится неактивным и наоборот. Необходимо обратить внимание на то, что строится одновременно полный набор всевозможных кластеров, поскольку в формуле (2.3) в гамильтонианах стоят суммы по всем связям и проекциям. В задачах роста кластеров следят, как правило, за ростом одного или ограниченного числа кластеров [17].

Не все кластеры из этого набора дают вклад в момент (2.3), а только кластеры с ненулевым шпуром. Таковыми являются кластеры с активным начальным узлом 0 и неактивными всеми остальными узлами. Необходимость обязательной дезактивации присоединенных узлов приводит к тому, что у представляющих $M_{2 n}$ связанных графов $2 n$ связей соединяют не более чем $n+1$ узлов, один из которых - начальный узел 0 . При этом одинаковые графы, построенные разными путями, являются разными членами суммы. 
После замены многократных связей однократными эта сумма сводится к сумме по базовым графам $g[3]$ - решеточным фигурам из связей:

$$
M_{2 n}=J^{2 n} \sum_{g} N(g) K_{2 n}(g),
$$

где $N(g)$ - число размешений без самопересечений данной фигуры на решетке, $J^{2 n} K_{2 n}$ вклад в момент, соответствуюший данной фигуре.

В пределе $d \rightarrow \infty[2,4]$ главный вклад в сумму $(2.4)$ дают корневые деревья с максимально возможным числом узлов $\left(n+1\right.$ для $\left.M_{2 n}\right)$. Но даже для них весовые множители $K_{2 n}$ не удается рассчитать. Однако в этом пределе можно указать границы величин моментов [2]. Верхнюю границу дают моменты решения уравнения Блюма-Хаббарда [16]

$$
F(t)=\exp \left\{-2 J^{2} Z \int_{0}^{t}\left(t-t^{\prime}\right) F\left(t^{\prime}\right) d t^{\prime}\right\}
$$

где в показателе экспоненты оставлен первый член кумулянтного разложения, $Z=2 d-$ число ближайших соседей. Нижнюю границу дает решение уравнения Резибуа-де Линера [15]. Более грубую нижнюю границу можно получить из решения того же уравнения (2.5) с уменьшенным в два раза коэффициентом перед интегралом.

Для прояснения указанного соотношения меж ду моментами сделаем в уравнении (2.5) замену переменной $J t=i \tau$ и посмотрим на него как на уравнение для производяшей функции числа корневых деревьев. С этой целью рассмотрим вспомогательную спиновую систему на решетке Бете с координационным числом $Z=\sigma+1$. Возможными фигурами в сумме (2.4) будут корневые деревья. Так как каждый добавленный узел должен быть дезактивирован, то деревья с максимально возможным числом узлов (которые в дальнейшем только и будут рассматриваться) построены с двойными связями. Понятно, что если присоединение новых узлов при построении дерева происходит последовательно, то и дезактивация узлов должна вьполняться последовательно, но в обратном направлении - от конца ветви к корню.

Возьмем деревья, у которых с корнем может быть связано не более одного соседнего узла (так называемые деревья с висячим корнем), и обозначим через $w_{1}(2 n)$ число таких деревьев, содержаших $n+1$ узлов и $n$ двойных связей. Предположим, что как построение, так и дезактивация различных ветвей дерева выполняются независимо. Тогда для числа деревьев имеем соотношение

$$
w_{1}(2 n)=\sum \frac{(2 n-2) !}{\left(2 n_{1}\right) !\left(2 n_{2}\right) ! \ldots\left(2 n_{\sigma}\right) !} w_{1}\left(2 n_{1}\right) w_{1}\left(2 n_{2}\right) \ldots w_{1}\left(2 n_{\sigma}\right),
$$

где суммирование выполняется по всем возможным распределениям $2 n-2$ связей среди $\sigma$ ветвей, растуших из ближайшего к корню узла. Введя производяшую функцию

$$
F_{1}(\tau)=\sum_{n=0}^{\infty} w_{1}(2 n) \frac{\tau^{2 n}}{(2 n) !}
$$


мы получаем из (2.6) уравнение

$$
F_{1}(\tau)=1+\int_{0}^{\tau} d \tau_{1} \int_{0}^{\tau_{1}} d \tau_{2}\left[F_{1}\left(\tau_{2}\right)\right]^{\sigma} .
$$

Используя $F_{1}(\tau)$, мы можем получить производящую функцию для числа деревьев, у которых корень имеет $Z$ соседей:

$$
F_{Z}(\tau)=\left[F_{1}(\tau)\right]^{Z}
$$

Чтобы перейти к пределу $Z \rightarrow \infty$, введем переменную $y=\tau \sqrt{Z}$ в (2.8). Тогда в этом пределе функция (2.9) переходит в функцию

$$
F_{\infty}(y)=\exp \left\{\int_{0}^{y} d y_{1} \int_{0}^{y_{1}} d y_{2} F_{\infty}\left(y_{2}\right)\right\} .
$$

Вернемся в $(2.10)$ к переменной $t=y /(i J \sqrt{Z})$. После этого мы получаем уравнение $(2.10)$ с показателем экспоненты, меньшим в два раза. Тем самым в случае (2.5) число деревьев с $n$ двойными связями будет больше в $(2)^{n}$ раз. Такое увеличение обусловлено тем, что в (2.4) в гамильтониане $\varkappa$ взаимодействие между каждой парой узельных спинов является суммой трех составляюших, содержаших $x$-, $y$ - и $z$ - компоненты спиновых операторов этих узлов. Одно и то же дерево может быть построено многими способами, отличаюшимися последовательностями проекций спиновых операторов. Если учесть, что коммутатор одноименных проекций обрашается в нуль, а выбор проекций операторов при дезактивации однозначен, то $2^{n}$ - максимально возможное значение числа способов для построения дерева из $n$ двойных связей. Случай равного единице коэффициента перед интегралом (2.10) соответствует выбору единственного набора операторов для каждого дерева. Реальный коэффициент находится между этими крайними значениями и зависит от структуры дерева и последовательности его построения, т.к. некоторые комбинации спиновых проекций дают нулевой результат при точном вычислении коммутаторов.

Перейдем теперь к анализу аналитических свойств автокорреляционной функции (2.1) системы размерности $d$. С этой целью будем использовать уравнения в безразмерной форме (2.7)-(2.10). Возврат к реальному времени осушествляется с помошью замены переменной $\tau=i t J p$. Согласие с уравнением $(2.5)$ достигается при $p=2$. Выбор другого значения для $p$ изменяет масштаб времени, но не изменяет свойств функций. Уравнение (2.8) эквивалентно дифференциальному уравнению второго порядка

$$
\ddot{F}_{1}(\tau)=\left[F_{1}(\tau)\right]^{\sigma},
$$

которое может быть решено в квадратурах. Его решение имеет ближайшие особые точки с координатами $\pm \tau_{b}$ на оси мнимого времени:

$$
\tau_{b}=\tau_{\infty} \frac{\Gamma\left(\frac{1}{2}-\frac{1}{Z}\right)}{\sqrt{\pi} \Gamma\left(1-\frac{1}{Z}\right)} \approx \tau_{\infty}\left(1+\frac{2 \ln 2}{Z}\right)
$$


где $\Gamma(x)$ - гамма-функция, $\tau_{\infty}=y_{\infty} / \sqrt{Z}$, а $y_{\infty}=\pi / \sqrt{2}$ - координата ближайшей особенности решения предельного уравнения (2.10) [16]

$$
F_{\infty}(y)=\frac{1}{\cos ^{2}\left(\frac{y}{\sqrt{2}}\right)}
$$

(для уравнения $(2.5) t_{\infty}=i \tau_{\infty} /(J \sqrt{2})$ ). В окрестности особенности для главной части решения находим

$$
F_{1}(\tau) \approx A_{1}\left(\tau_{b}-\tau\right)^{-\frac{2}{k}},
$$

где

$$
k=\sigma-1=Z-2, \quad A_{1}=\left\{\frac{2 Z}{k^{2}}\right\}^{\frac{1}{k}} .
$$

Тем самым мы получаем, что в приближении Бете при уменьшении $d$ у автокорреляционных функций сохраняются особые точки на оси мнимого времени, хотя их координата увеличивается. Напомним, что при выводе уравнения (2.11) мы сохранили в сумме (2.4) только деревья с максимальным числом узлов, построенные с двойными связями. В приближении Бете мы учли важное ограничение, заключаюшееся в том, что при конечном $d$ из каждого узла дерева вырастает не более $\sigma$ ветвей. Влияние другого ограничения - запрета пересечения ветвей этих деревьев (эффекта исключенного объема) - будет рассмотрено в следуюших разделах. В конце этого раздела для сравнения приведем аналогичные результаты приближения Бете для деревьев, построенных с одиночными связями. Известны два типа таких деревьев. В модели Идена [7], как и у нас, деревья различаются по последовательности присоединения связей, т.е. по способу построения, тогда как в теории разветвленных молекул $[5,9,10]$ и перколяции $[5]$ деревья различаются только по топологии и способу размешения на решетке.

Начнем с модели Идена. Введем производяшую функцию числа $v_{n}$ таких деревьев с висячим корнем

$$
E_{1}(x)=\sum_{n=0}^{\infty} v_{n} \frac{x^{n}}{n !} .
$$

Эта функция удовлетворяет уравнению

$$
E_{1}(x)=1+\int_{0}^{x} d x_{1}\left[E_{1}\left(x_{1}\right)\right]^{\sigma},
$$

поскольку соотношение для $v_{n}$ получается из (2.6) заменой всех чисел $2 n_{i}$ на $n_{i}$. Уравнение (2.16) эквивалентно дифференциальному уравнению

$$
\frac{d E_{1}(x)}{d x}=\left[E_{1}(x)\right]^{\sigma},
$$

которое имеет решение

$$
E_{1}(x)=(1-k x)^{-\frac{1}{k}} .
$$


Результат (2.18) совпадает с соответствуюшим результатом работы [7], полученным другим способом.

Для обычных решеточных деревьев $[5,6,9,10]$ с висячим корнем производяшая функция

$$
G_{1}(x)=\sum_{n=0}^{\infty} T_{n} x^{n}
$$

где $T_{n}$ - число таких деревьев, удовлетворяет алгебраическому уравнению

$$
G_{1}(x)=1+x\left[G_{1}(x)\right]^{\sigma},
$$

решение которого имеет особенность при

$$
x_{b}=\frac{\left(1-\frac{1}{\sigma}\right)^{k}}{\sigma}
$$

В особой точке

$$
G_{1}\left(x_{b}\right)=\frac{\sigma}{\sigma-1}=\left(x_{b} \sigma\right)^{-\frac{1}{k}}
$$

а вблизи нее

$$
G_{1}(x) \approx \frac{\sigma}{k}-\left[2 \sigma k^{-3} \frac{x_{b}-x}{x_{b}}\right]^{\frac{1}{2}}
$$

\section{3. ПОПРАВКИ К ПРОИЗВОДЯШЕЙ ФУНКЦИИ ОТ ПЕРЕСЕЧЕНИЙ ВЕТВЕЙ ДЕРЕВЬЕВ}

Перенесем теперь деревья, построенные на решетке Бете, на простую гиперкубическую решетку. Часть деревьев удается разместить только при повторном использовании одних и тех же узлов и связей, т.е. при допушении самопересечений. Другая часть деревьев может быть размешена без самопересечений. Простейшая петля, которая образуется при пересечении ветвей, - это квадрат. Дерево из $n$ двойных связей, каждая из которых располагается в новом измерении, может быть размешено на решетке $d^{n}$ способами (с точностью до численного коэффициента). Поскольку у квадрата направления противоположных сторон совпадают, то дерево, ветви которого образовали квадрат, можно разместить меньшим в $d^{2}$ числом способов. Понятно, что у петли, образованной бо́льшим числом звеньев, потери еше больше $[8,9]$.

Найдем первую поправку к производяшей функции для деревьев с висячим корнем от простейшего пересечения ветвей. Для ее записи преобразуем уравнение (2.8). Первоначальная его форма отражает тот факт, что все деревья могут быть рассортированы по числу ответвлений из ближайшего к корню узла. Иначе деревья могут быть рассортированы по числу связей вдоль некоторого направления, выбранного на решетке Бете. Рассмотрим сразу общий случай, когда цепочка из $n$ звеньев вдоль выбранного направления может заканчиваться не только свободным концом, но иметь на конще некоторый 
фрагмент (например петлю), описываемый функцией $f(\tau)$. Производящая функция для деревьев, включаюших такую цепочку, имеет вид

$$
\begin{gathered}
L_{n}(\tau, f)=\int_{0}^{\tau} d \tau_{1}^{\prime} \int_{0}^{\tau_{1}^{\prime}} d \tau_{1}\left[F_{1}\left(\tau_{1}\right)\right]^{k} \int_{0}^{\tau_{1}} d \tau_{2}^{\prime} \int_{0}^{\tau_{2}^{\prime}} d \tau_{2}\left[F_{1}\left(\tau_{2}\right)\right]^{k} \times \cdots \\
\cdots \times \int_{0}^{\tau_{n-1}} d \tau_{n}^{\prime} \int_{0}^{\tau_{n}^{\prime}} d \tau_{n}\left[F_{1}\left(\tau_{n}\right)\right]^{k} f\left(\tau_{n}\right), \\
L_{0}(\tau, f)=f(\tau) .
\end{gathered}
$$

Для наглядности представим эту функцию диаграммой

$$
\underset{\tau}{\rightleftharpoons} \underset{\tau_{1}}{\rightleftharpoons} \tau_{\tau_{2}} \ldots \underset{\tau_{n-1}}{\rightleftharpoons} \mathrm{O}\left\{f\left(\tau_{n}\right)\right\}
$$

Двойной линией в диаграмме обозначена занятая связь, которой соответствует двойной интеграл в $(3.1)$, функции $\left[F_{1}\left(\tau_{p}\right)\right]^{k}$ в узле $p$ соответствует пустой кружок, а корнюкружок с точкой.

Вводя формальный параметр $\theta$ для задания числа звеньев и суммируя вклады (3.1) для цепочек разной длины вдоль выбранного направления, получаем соответствуюшую производяшую функцию

$$
L(\theta, \tau, f)=\sum_{n=0}^{\infty} \theta^{n} L_{n}(\tau, f),
$$

удовлетворяюшую уравнению

$$
L(\theta, \tau, f)=f(\tau)+\theta \int_{0}^{\tau} d \tau_{1}^{\prime} \int_{0}^{\tau_{1}^{\prime}} d \tau_{1}\left[F_{1}\left(\tau_{1}\right)\right]^{k} L\left(\theta, \tau_{1}, f\right) .
$$

Легко видеть, что при $\theta=1, f(\tau)=1$ уравнение (3.4) переходит в уравнение (2.8), поскольку $L(1, \tau, 1)=F_{1}(\tau)$. В этом случае ряд (3.3) - итерационный ряд уравнения (2.8), полученный последовательными итерациями вдоль выбранного направления.

Подобным путем можно получить производящую функцию для деревьев, содержаших другие фигуры из цепочек. Так, например, три возможных варианта пересечения двух ветвей, выходяших из ближайшего к корню узла, могут быть представлены следуюшими тремя диаграммами:
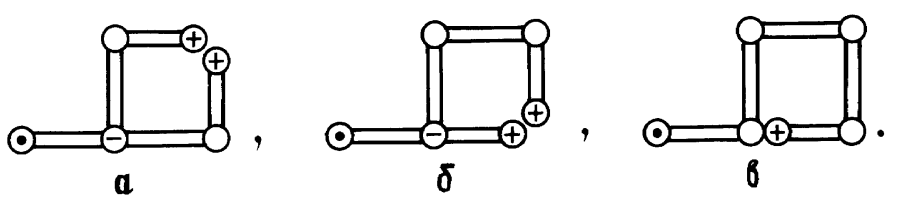

Кружок с плюсом и кружок с минусом обозначают, что показатель степени, в которую возводится функция $F_{1}(\tau)$, для данного узла равен соответственно $k+1$ и $k-1$, а два соседних кружка в вершине квадрата обозначают пересечение. 
С помощью функции (3.1), взятой при $f(\tau)=F_{1}(\tau)$, явные выражения для диаграмм (3.5) можно записать в следуюшем виде:

$$
\int_{0}^{\tau} d \tau_{1}^{\prime} \int_{0}^{\tau_{1}^{\prime}} d \tau_{1}\left[F_{1}\left(\tau_{1}\right)\right]^{k-1} L_{a}\left(\tau_{1}, F_{1}\right) L_{b}\left(\tau_{1}, F_{1}\right)
$$

Под интегралом в (3.6) стоят следуюшие произведения:

1) в случае $(3.5 \mathrm{a})\left[L_{2}\left(\tau_{1}, F_{1}\right)\right]^{2}$,

$2)$ в случае $(3.5 б) L_{3}\left(\tau_{1}, F_{1}\right) L_{1}\left(\tau_{1}, F_{1}\right)$,

$3)$ в случае $(3.5 \mathrm{~B}) L_{4}\left(\tau_{1}, F_{1}\right) L_{0}\left(\tau_{1}, F_{1}\right)$.

Для каждого конкретного квадрата на решетке будут одна диаграмма (3.5а) и по две диаграммы (3.5б) и (3.5в), отличаюшиеся перестановкой длинной и короткой цепочек, образующих квадрат. Обозначим сумму вкладов от одной петли из $q$ звеньев через $P_{q}(\tau)$. Для квадрата имеем

$$
P_{4}(\tau)=\left[L_{2}\left(\tau, F_{1}\right)\right]^{2}+2 L_{3}\left(\tau, F_{1}\right) L_{1}\left(\tau, F_{1}\right)+2 L_{4}\left(\tau, F_{1}\right) L_{0}\left(\tau, F_{1}\right) .
$$

Эту сумму можно выразить через вспомогательную функцию $(3.3)$, взятую при $f(\tau)=$ $F_{1}(\tau)$, для которой введем свое обозначение

$$
\Phi(\theta, \tau) \equiv L\left(\theta, \tau, F_{1}\right)
$$

Возведя сумму (3.3) во вторую степень, легко проверить, что сумма (3.7) есть коэффициент перед $\theta^{4}$ в $[\Phi(\theta, \tau)]^{2}$. Уравнение $(3.4)$ для введенной функции удобно переписать в виде дифференциального уравнения второго порядка

$$
\ddot{\Phi}(\theta, \tau)=\left[F_{1}(\tau)\right]^{\sigma}+\theta\left[F_{1}(\tau)\right]^{k} \Phi(\theta, \tau)
$$

с начальными условиями $\Phi(\theta, 0)=1$ и $\dot{\Phi}(\theta, 0)=0$.

Петля с пересечением может располагаться на дереве на любом расстоянии от корня и вдоль любого направления. Для петли, присоединенной к корню цепью из $n$ звеньев, в выражении (3.1) следует взять

$$
f(\tau)=\int_{0}^{\tau} d \tau_{1}^{\prime} \int_{0}^{\tau_{1}^{\prime}} d \tau_{1}\left[F_{1}\left(\tau_{1}\right)\right]^{k-1} P_{q}\left(\tau_{1}\right) .
$$

Вследствие тож дественности узлов и направлений суммирование по направлениям сводится к домножению вклада (3.1) от цепей из $n$ звеньев на $\sigma^{n}$. После этого суммирование по длинам цепей, ведущих к петле, может быть выполнено с помощью уравнений (3.3), (3.4) с $\theta=\sigma$ и функцией $f(\tau)$, заданной выражением (3.9).

С использованием функции $\Phi(\theta, \tau)$ искомый результат $R_{4}(\tau)$ может быть получен как коэффицциент перед $\theta^{4}$ в разложении функции $R(\theta, \tau) \equiv L(\sigma, \tau, f)$, являюшейся решением уравнения (3.4) при $\theta=\sigma$ и

$$
f(\tau)=\int_{0}^{\tau} d \tau_{1}^{\prime} \int_{0}^{\tau_{1}^{\prime}} d \tau_{1}\left[F_{1}\left(\tau_{1}\right)\right]^{k-1}\left[\Phi\left(\theta, \tau_{1}\right)\right]^{2}
$$


Уравнение (3.4) для $R(\theta, \tau)$ при функции $f$, заданной формулой (3.10), удобно переписать в виде дифференциального уравнения второго порядка

$$
\ddot{R}(\theta, \tau)=\left[F_{1}(\tau)\right]^{k-1}[\Phi(\theta, \tau)]^{2}+\sigma\left[F_{1}(\tau)\right]^{k} R(\theta, \tau)
$$

с начальными условиями $R(\theta, 0)=\dot{R}(\theta, 0)=0$.

Наконец, чтобы получить окончательное выражение для поправки от простейшего пересечения ветвей деревьев, результат $R_{4}(\tau)$ следует просуммировать по всем положениям квадрата у данного узла. Число таких положений составляет

$$
N_{4}=\frac{k^{2}}{2}
$$

Мы исключили связь, подводяшую от корня к данному узлу, из числа доступных, поэтому наше число меншше чем $Z(Z-2) / 2[9]$.

Подобным путем можно получать результаты для других, более сложных фигур [9], образующихся при пересечении ветвей деревьев. В частности, результат для вклада в производящую функцию от пересечения ветвей в виде петли из шести звеньев получается как коэффишиент перед $\theta^{6}$ в разложении $R(\theta, \tau)$. Число таких петель у одного узла составляет

$$
N_{6}=(\sigma-1)\left[(\sigma-2)(2 \sigma-3)-\frac{1}{2}\right] .
$$

При расчете поправок, имеюших следуюший порядок малости по $1 / \sigma$, помимо добавления главного вклада от сложных фигур, следует учесть и поправки соответствующего порядка к главному вкладу, содержашиеся в простых фигурах. Так, например, запись $P_{4}(\tau)$ в виде (3.7) точна в главном порядке $\sigma^{-2}$, когда при пересечении совпадают два узла. Если же в месте пересечения накладываются две связи:
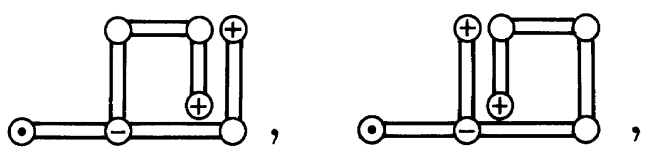

то в $P_{4}(\tau)$ этот вклад учитывается дважды. Поэтому с точностью $1 / \sigma^{3}$ из суммы $(3.7)$ следует вычесть

$$
2 L_{3}\left(\tau, F_{1}\right) L_{2}\left(\tau, F_{1}\right)+2 L_{4}\left(\tau, F_{1}\right) L_{1}\left(\tau, F_{1}\right) .
$$

Естественно, не следует забывать и поправки от (3.12). Описанная процедура иллюстрируется в приложении на примере решеточных фигур, изученных ранее другими методами $[8-10]$. 


\section{4. РАСЧЕТ КООР ДИНАТ ОСОБЫХ ТОЧЕК}

4.1. Деревья, построенные с одинарными связями. Полученные формулы сначала применим к более простому случаю деревьев в модели Идена. Как легко заключить из сравнения выражений (2.16) и (2.17) с выражениями (2.8) и (2.11), необходимые для этого случая формулы получаются из формул предыдушего раздела после замены двойных производных и интегралов на однократные. Решая уравнения, полученные таким путем из уравнений (3.8) и (3.11), находим

$$
\begin{aligned}
& R(\theta, x)=\frac{1}{k} y^{-\frac{\sigma}{k}} \int_{y}^{1} y_{1}^{\frac{2}{k}}\left[\Phi\left(\theta, x_{1}\right)\right]^{2} d y_{1}, \\
& \Phi(\theta, x)=\frac{y^{-\frac{1}{k}}-\theta y^{-\frac{\theta}{k}}}{1-\theta}
\end{aligned}
$$

где $y=1-k x$. После вычисления интеграла в (4.1) получаем

$$
R(\theta, x)=\left\{1-y-2 \theta \frac{1-y^{1+\frac{1-\theta}{k}}}{1+\frac{1-\theta}{k}}+\theta^{2} \frac{1-y^{1+2 \frac{1-\theta}{k}}}{1+2 \frac{1-\theta}{k}}\right\} \frac{1}{k(1-\theta)^{2} y^{\frac{\sigma}{k}}} .
$$

Коэффициент $R_{q}(x)$ при $\theta^{q}$ разложения (4.3) дает производящую функцию для числа деревьев с одним пересечением ветвей в виде простой петли из $q$ звеньев. Для интересуюшего нас сейчас квадрата находим

$$
\begin{aligned}
& R_{4}(x)\left(k y^{\frac{\sigma}{k}}\right)=5(1-y)-2 k(1+k)^{-4}\left(4 k^{3}+15 k^{2}+20 k+10\right)\left(1-y^{1+\frac{1}{k}}\right)+ \\
& \quad+k(2+k)^{-3}\left(3 k^{2}+16 k+24\right)\left(1-y^{1+\frac{2}{k}}\right)-2(1+k)^{-3}\left(3 k^{2}+8 k+6\right) y^{1+\frac{1}{k}} \ln y+ \\
& \quad+4(2+k)^{-2}(k+3) y^{1+\frac{2}{k}} \ln y+(1+k)^{-2}\left(2+\frac{3}{k}\right) y^{1+\frac{1}{k}} \ln ^{2} y- \\
& \quad-2(2+k)^{-1} k^{-1} y^{1+\frac{2}{k}} \ln ^{2} y-(1+k)^{-1} k^{-2} 3^{-1} y^{1+\frac{1}{k}} \ln ^{3} y
\end{aligned}
$$

Домножим этот результат на число положений квадрата у данного узла $N_{4}(3.12)$ и вычтем из (2.18). Получаем, что для производящей функции деревьев с висячим корнем с первой поправкой на пересечение ветвей имеем

$$
E_{1}(x)=(1-k x)^{-\frac{1}{k}}-\frac{k^{2} R_{4}(x)}{2}+\cdots .
$$

На основании двух членов разложения (4.5) определим координату $x_{c}$ особой точки. Эта координата входит в асимптотическую формулу числа деревьев. В работе [9] для решеточных фигур предложена процедура вычисления аналогичного параметра посредством нахождения числа кластеров большого размера. Нахождение этих чисел сама по себе задача сложная. Как мы сейчас покажем, ее можно избежать и, используя идею метода работы [9], определить искомый параметр $x_{c}$ непосредственно по производяшей функции. В приложении этот подход проверен на решеточных фигурах, уже изученных ранее $[9,10]$. Сейчас применим его к деревьям в модели Идена (4.5). 
В приближении Бете $(2.18)$ было $x_{c}=x_{b}=1 / k$. Запрет на пересечение ветвей увеличивает координату, т.е.

$$
x_{c}=x_{b}+\delta x_{c} .
$$

Будем рассматривать производящую функцию как функцию двух параметров $x$ и $x_{c}$ и разложим ее по малому параметру $\delta x_{c}$ :

$$
E_{1}\left(x, x_{c}\right)=E_{1}\left(x, x_{b}\right)+\left.\delta x_{c} \frac{\partial E_{1}\left(x, x_{c}\right)}{\partial x_{c}}\right|_{x_{c}=x_{b}}+\cdots
$$

Сравнивая (4.5) с (4.6) и полагая

$$
\left.\frac{\partial E_{1}\left(x, x_{c}\right)}{\partial x_{c}}\right|_{x_{c}=x_{b}}=\frac{\partial E_{1}\left(x, x_{b}\right)}{\partial x_{b}}=-k x(1-k x)^{-\frac{\sigma}{k}}
$$

находим

$$
\delta x_{c}=\left\{R_{4}(x) \frac{k y^{\frac{\sigma}{k}}}{2 x}\right\}_{x=x_{b}}=\left[8 \sigma^{3}+7 \sigma^{2}+4 \sigma+1\right] k \sigma^{-4} Z^{-3} .
$$

Отсюда следует, что в главном порядке по $1 / \sigma$

$$
x_{c}=x_{b}\left(1+8 \sigma^{-2}\right)
$$

4.2. Деревья, построенные с двойными связями. Для деревьев с двойными связями (в отличие от одинарных) уравнения, полученные в третьем разделе, в аналитическом виде решить не удается. Однако интересуюшая нас поправка к координате особой точки определяется поведением функций в окрестности этой точки, где главные части решений уравнений могут быть получены достаточно просто.

Подставив (2.14) в уравнение (3.11), находим для главной части его решения вблизи особенности

$$
R(\theta, \tau) \approx C\left(1-\frac{\tau}{\tau_{b}}\right)^{-1-\frac{2}{k}}
$$

где

$$
C=\frac{\tau_{b}}{3+\frac{4}{k}} \int_{0}^{\tau_{b}}\left(1-\frac{\tau}{\tau_{b}}\right)^{2+\frac{2}{k}}\left[F_{1}(\tau)\right]^{k-1}[\Phi(\theta, \tau)]^{2} d \tau
$$

Поскольку функцию $\Phi(\theta, \tau)$ найти не удается, то для расчета поправки $R_{4}$ от простейшего пересечения ветвей в виде квадрата подставим вместо $\Phi(\theta, \tau)$ в $(4.9)$ функцию $P_{4}(\tau)$ из (3.7). Затем перейдем в $(4.9)$ и в многократных интегралах в $(3.7)$ к безразмерным переменным $\varphi=\pi \tau /\left(2 \tau_{b}\right)$. В результате получаем

$$
R_{4}=\frac{\tau_{b}^{10}}{3+\frac{4}{k}}\left(\frac{\pi}{2}\right)^{11} \int_{0}^{\frac{\pi}{2}}\left(\frac{\pi}{2}-\varphi\right)^{2+\frac{2}{k}}\left[F_{1}\left(2 \varphi \frac{\tau_{b}}{\pi}\right)\right]^{k-1} P_{4}(\varphi) d \varphi
$$

При больших $Z$ порядок величины коэффициента перед интегралом согласно (2.12) равен $Z^{-5}$. Сам интеграл имеет порядок $Z^{0}$. Для нахождения его главной части в пределе $Z \rightarrow \infty$ в подынтегральном выражении возьмем для функций $F_{1}^{k}$ и $F_{1}^{k \mp 1}$ предельную 
форму $(2.13)$ и заменим $(\pi / 2-\varphi)^{2+2 / k}$ на $(\pi / 2-\varphi)^{2}$. Вычислив интеграл, находим в главном порядке по $Z$, что

$$
R_{4}=\frac{2^{6} \cdot 5.867}{3 \pi Z^{5}}
$$

Подставим (4.10) в (4.8), домножим на $N_{4}(3.12)$ и вычтем результат из (2.14). После чего для главной части производяшей функшии для деревьев с висячим корнем, построенных с двойными связями, получаем искомое выражение с поправкой:

$$
F_{1}(\tau)=A_{1}\left(\tau_{b}-\tau\right)^{-\frac{2}{k}}-\frac{1}{2} k^{2} R_{4}\left(\tau_{b}-\tau\right)^{-1-\frac{2}{k}} \tau_{b}^{1+\frac{2}{k}}+\cdots .
$$

Сравнив поправочный член этого выражения с поправочным членом

$$
\left.\delta \tau_{0} \frac{\partial F_{1}(\tau)}{\partial \tau_{0}}\right|_{\tau_{0}=\tau_{b}}=\delta \tau_{0}\left(-\frac{2}{k}\right) A_{1}\left(\tau_{b}-\tau\right)^{-1-\frac{2}{k}}
$$

аналогичного (4.6) разложения функции $F_{1}\left(\tau, \tau_{0}\right)$ по малому сдвигу $\delta \tau_{0}$ координаты особой точки, находим

$$
\delta \tau_{0}=\frac{k^{3} R_{4} \tau_{b}^{1+\frac{2}{k}}}{4 A_{1}} .
$$

Сохранив в этом выражении главный порядок по $1 / \sigma$, для координаты особой точки автокорреляционной функции в приближении деревьев с первой поправкой от пересечения ветвей получаем

$$
\tau_{b}+\delta \tau_{0}=\tau_{b}\left(1+9.96 \sigma^{-2}\right) .
$$

В асимптотической формуле (4.12) подразумевается, что $d \gg 1$. Однако уже при $d=3$ можно считать $1 / \sigma$ малой величиной и получить $\tau_{0} \approx 1.4 \tau_{b}$. В работе [4] для этого же случая методом компютерного моделирования была получена оценка $\tau_{0} \approx 1.2 \tau_{b}$. Занижение результата, по-видимому, следует отнести на счет недостаточного для полного проявления эффекта исключенного объема размера деревьев, доступных для расчета.

\section{5. ОБСУЖДЕНИЕ}

Выше мы рассмотрели производяшие функции деревьев на решетках большой размерности при учете пересечений ветвей и получили результаты для координат особых точек с поправкой $1 / \sigma^{2}$ для автокорреляционной функции (4.12) (деревья из двойных связей) и модели Идена (деревья из одинарных связей) (4.7). Сравнивая эти результаты между собой и с результатом (П.8) (см. приложение) для решеточных фигур, видим, что в трех случаях координаты особых точек увеличиваются при уменьшении $d$ схожим образом. Никаких качественных различий, позволяюших усомниться в сушествовании у автокорреляционной функции особых точек на конечном расстоянии, не обнаружено.

Для того чтобы сделать окончательное заключение, предстоит еще учесть вклад в моменты (2.4) от решеточных фигур с петлями и многократным взаимодействием одних и тех же спинов. Однако уже на основании выполненного исследования следует ожидать, 
что добавление новых локальных поправок не изменит радикальным образом свойства больших деревьев, определяюших положение ближайших особых точек автокоррелящионной функции спиновой системы большой размерности. Данное предположение оправдано и тем, что наличие петель у больших разветвленных молекул в теории полимеров не приводит к существенным изменениям асимптотических свойств [18].

Остается нерешенной известная для задач такого типа проблема неперестановочности пределов $d \rightarrow \infty$ и $n \rightarrow \infty$ [11]. Если величину $d$ оставить конечной, а размер кластера увеличивать, то при $n>d$ учтенные поправочные члены могут перестать быть главными. Результат для координаты особой точки автокорреляционной функции тогда изменится, но, как мы ожидаем по аналогии с другими критическими явлениями, сама особенность должна сохраниться. В подтверждение этого сошлемся также на экспериментальные данные. Дело в том, что одним из следствий наличия указанных особенностей корреляционных функций на оси мнимого времени являются экспоненциальные высокочастотные асимптотики их спектров. Экспериментально наблюдаемые “крылья" спектров оказываются близкими по форме к экспоненциальным (см. соответствующий анализ в работах $[2,19,20])$. Основная часть экспериментов выполнена методом ядерного магнитного резонанса на системах с анизотропным диполь-дипольным взаимодействием. При обобшении на системы с анизотропным взаимодействием [19-21] усложняются уравнения и формулы, но сами выводы качественно не изменяются.

Благодарности. Автор признателен М. А. Попову за помощь при расчетах на компютере. Работа выполнена при финансовой поддержке Российского фонда фундаментальных исследований (проект № 99-02-18214).

\section{ПРИЛОЖКНИЕ}

Ниже предложенньй подход к нахождению координаты особой точки производящей функции будет проверен на решеточных фигурах, $1 / \sigma$-разложение для которых хорошо известно $[9,10]$. Ограничимся двумя первыми членами порядка $\sigma^{-2}$ и $\sigma^{-3}$.

Рассмотрим поправку к (2.19) от пересечения ветвей деревьев. Поскольку слагаемые в (3.7) и (3.15), различающиеся положением места пересечения на квадрате, теперь одинаковы, то вклад от квадрата составляет

$$
P_{4}(x)=5 x^{4}\left[G_{1}(x)\right]^{4 k+2}-4 x^{5}\left[G_{1}(x)\right]^{5 k+2} .
$$

Аналогично находим вклад от пересечения ветвей деревьев в виде петли из шести звеньев:

$$
P_{6}(x)=7 x^{6}\left[G_{1}(x)\right]^{6 k+2}
$$

Если рассматривать не только деревья, но и фигуры с петлями [9], то к производяшей функции следует добавить соответствующий член. Настоящие петли отличаются от пересечения ветвей деревьев отсутствием свободных концов. Поэтому вклады для них получаются из $P_{4}(x)$ (первое слагаемое (П.1)) и $P_{6}(x)$ (П.2) делением соответственно на $5\left[G_{1}(x)\right]^{\sigma+1}$ и $7\left[G_{1}(x)\right]^{\sigma+1}$. Эти вклады не вычитаются из $G_{1}(x)$, а прибавляются.

5 Теоретическая и математическая физика, т. 123, № 1, 2000 г. 
Для обычных деревьев аналогом уравнения (3.4) является алгебраическое уравнение, которое при $\theta=\sigma$ имеет решение

$$
L(\sigma, x, f)=\frac{f(x)}{1-\sigma x\left[G_{1}(x)\right]^{k}}
$$

Используя результаты (П.1)-(П.3), для производящей функции в случае фигур с висячим корнем получаем при учете поправок от пересечения ветвей деревьев и от настоящих петель

$$
G_{1}(x)-\frac{B(x)\left[G_{1}(x)\right]^{\sigma}}{1-\sigma x\left[G_{1}(x)\right]^{k}}+\cdots
$$

где

$$
\begin{aligned}
B(x)= & N_{4} x^{5}\left[G_{1}(x)\right]^{4 k}\left[5-4 x\left[G_{1}(x)\right]^{k}-\left[G_{1}(x)\right]^{-\sigma-1}\right]+ \\
& +N_{6} x^{7}\left[G_{1}(x)\right]^{6 k}\left[7-\left[G_{1}(x)\right]^{\sigma-1}\right] .
\end{aligned}
$$

Для определения точки $x_{c}$ на основании (П.4) представим это выражение в виде аналогичного (4.6) разложения по малому сдвигу $\delta x_{c}$. Действительно, согласно $(2.20)$ имеem

$$
\frac{d G_{1}(x)}{d x}=\frac{\left[G_{1}(x)\right]^{\sigma}}{1-\sigma x\left[G_{1}(x)\right]^{k}}
$$

тогда как согласно (2.23) вблизи особой точки

$$
\frac{\partial G_{1}\left(x, x_{c}\right)}{\partial x} \approx-\frac{\partial G_{1}\left(x, x_{c}\right)}{\partial x_{c}}
$$

С учетом (П.5) и (П.6) из (П.4) получаем

$$
\delta x_{c}=B\left(x_{b}\right)
$$

В соответствии с $(2.22)$ в особой точке находим, что

$$
\left[G_{1}\left(x_{b}\right)\right]^{q k}=\left(x_{b} \sigma\right)^{-q}, \quad\left[G_{1}\left(x_{b}\right)\right]^{-\sigma-1}=\left(1-\frac{1}{\sigma}\right)^{\sigma+1} \approx \frac{1-\frac{3}{2 \sigma}}{e}
$$

Подставив эти значения и значения $N_{4}$ и $N_{6}$ в (П.7), окончательно получаем при сохранении членов порядка $\sigma^{-2}$ и $\sigma^{-3}$ формулу

$$
x_{c}=x_{b}\left[1+\frac{1}{\sigma^{2}}\left(\frac{5}{2}-\frac{1}{2 e}\right)+\frac{1}{\sigma^{3}}\left(7-\frac{1}{4 e}\right)\right],
$$

которая совпадает с соответствующим результатом работы [9]. 
ОСОБЫЕ ТОЧКИ ЗАВИСЯЩИХ ОТ ВРЕМЕНИ КОРРЕЛЯЦИОННЫХ ФУНКЦИЙ. .131

\section{Список литературы}

[1] H. Araki. Commun. Math. Phys. 1969. V. 14. P. 120.

[2] B.Е. Зобов. ТМФ. 1988. Т. 77. С. 426.

[3] T. Morita. J. Math. Phys. 1971. V. 12. P. 2062.

[4] B. Е. Зобов, М. А. Попов. ТМФ. 1997. Т. 112. С. 479.

[5] M. E. Fisher, J. W. Essam. J. Math. Phys. 1961. V. 2. P. 609.

[6] D. S. Gaunt, M. F. Sykes, G. M. Torrie, S. G. Whittington. J. Phys. A. 1982. V. 15. P. 3209.

[7] J. Vannimenus, B. Nickel, V. Hakim. Phys. Rev. B. 1984. V. 30. P. 391.

[8] M. E. Fisher, D.S. Gaunt. Phys. Rev. A. 1964. V. 133. P. 224.

[9] A. B. Harris. Phys. Rev. B. 1982. V. 26. P. 337.

[10] P. J. Peard, D. S. Gaunt. J. Phys. A. 1995. V. 28. P. 6109.

[11] R. Friedberg. Ann. Phys. (NY). 1986. V. 171. P. 321.

[12] W. M. Zheng. Phys. Rev. A. 1987. V. 39. P. 4904.

[13] R. Bersohn, T. P. Das. Phys. Rev. 1963. V. 130. P. 98.

[14] S. J, Knak Jensen, E. Kjaersgaard Hansen. Phys. Rev. B. 1973. V. 7. P. 2910.

[15] P. Resibois, M. De Leener. Phys. Rev. 1966. V. 152. P. 305.

[16] M. Blume, J. Hubbard. Phys. Rev. B. 1970. V. 1. P. 3815.

[17] Z. Alexandrowicz. Phys. Rev. Lett. 1985. V. 54. P. 1420.

[18] S. G. Whittington, C. M. Torrie, D. S. Gaunt. J. Phys. A. 1983. V. 16. P. 1695.

[19] В. Е. Зобов, А. А. Лундин. ЖЭТФ. 1994. Т. 106. С. 1097.

[20] В.Е. Зобов, М. А. Попов, Ю. Н. Иванов, А. И. Лившич. ЖЭТФ. 1999. Т. 115. С. 285.

[21] B. Е. Зобов. ТМФ. 1990. Т. 84. C. 111.

Поступила в редакцию 14.VII.1999 г., после доработки 30.IX.1999 г. 